\title{
Overexpression of LncRNA SNHGI Were Suitable for Oncolytic Adenoviruse HIOI Therapy in Oral Squamous-Cell Carcinoma
}

This article was published in the following Dove Press journal: OncoTargets and Therapy

\author{
Xin Wang ${ }^{1, *}$ \\ Song Yang ${ }^{1} *$ \\ Xuechao $\mathrm{Lv}^{2}$ \\ Lina Wang ${ }^{3}$ \\ Chunmei $\mathrm{Li}(\mathbb{D})^{4}$ \\ 'Department of Plastic Maxillofacial \\ Surgery, Heilongjiang Provincial Hospital, \\ Harbin I50036, Heilongjiang, People's \\ Republic of China; ${ }^{2}$ Department of \\ Pediatric Dentistry, School of \\ Stomatology, Harbin Medical University, \\ Harbin I5000I, Heilongiiang, People's \\ Republic of China; ${ }^{3}$ Department of Oral \\ and Maxillofacial Surgery, Second \\ Affiliated Hospital of Harbin Medical \\ University, Harbin I50086, Heilongjiang, \\ People's Republic of China; ${ }^{4}$ Department \\ of Outpatient Stomatology, Heilongjiang \\ Provincial Hospital, Harbin I50036, \\ Heilongjiang, People's Republic of China \\ *These authors contributed equally to \\ this work
}

Background: As the most prevalent type of head and neck cancer, oral squamous-cell carcinoma (OSCC) accounts for nearly $90 \%$ of all oral cancer cases. Despite great progress having been made in the diagnosis and treatment of OSCC recently, the survival rate of OSCC patients has not risen remarkably. Chemotherapy is commonly used for OSCC treatment; however, the emergence of chemoresistance limits its long-term curative effect. Therefore, identifying effective biomarkers and molecular mechanisms is essential to the development of therapeutic strategies for OSCC.

Methods: qRT-PCR assays were performed to detect SNHG1 expression in OSCC tissue and cells, and CCK8 assays and animal experiments used to examine cell proliferation. In addition, $\mathrm{CCK} 8$ assays were used to detect $\mathrm{IC}_{50}$ values of cisplatin, $5 \mathrm{Fu}$, Dox, and oncolytic adenovirus H101.

Results: We found that SNHG1 was overexpressed in OSCC tissue and cells and was associated with OSCC progression. In addition, knockdown of SNHG1 suppressed cell proliferation in vitro and in vivo. Importantly, we found that oncolytic adenovirus H101 showed better antitumor effects in OSCC with high SNHG1 expression, and chemotherapy showed worse anti-tumor effects in OSCC with high SNHG1 expression.

Conclusion: $S N H G 1$ can act as a diagnostic biomarker for OSCC, and may be a biomarker for treatment options.

Keywords: SNHG1, H101, OSCC

\section{Introduction}

As the most prevalent type of head and neck cancer, oral squamous-cell carcinoma (OSCC), accounts for nearly $90 \%$ of all oral cancer cases. ${ }^{1-3}$ Despite great progress having been made in the diagnosis and treatment of OSCC recently, the survival rate of OSCC patients has not risen remarkably. ${ }^{4-6}$ Therefore, identifying effective biomarkers and molecular mechanisms is essential to the development of therapeutic strategies for OSCC.

Lacking protein-coding capacity, lncRNAs are a class of regulatory transcripts $>200$ nucleotides. Early research revealed lncRNAs played a significant role in tumorigenesis via multiple mechanisms, such as with microRNAs or proteins. For example, $D A N C R$ lncRNA promotes tumor progression and cancer-stemness features in osteosarcoma by upregulating Axl via miR33a-5p inhibition. ${ }^{7} S N H G 3$ lncRNA induces epithelial-mesenchymal transition and sorafenib resistance by modulating the miR128-CD151 pathway in hepatocellular carcinoma. ${ }^{8} C R N D E$
Correspondence: Chunmei Li Department of Outpatient Stomatology, Heilongjiang Provincial Hospital, Harbin 150036, Heilongjiang, People's Republic of China

Tel $+86451-8713-1023$

Email lichunmei28I4@I63.com 
IncRNA promotes colorectal cancer-cell proliferation and chemoresistance via miR181a-5p-mediated regulation of Wnt- $\beta$-catenin signaling. ${ }^{9}$ Therefore, study of IncRNAs may be of remarkable value in understanding the occurrence and development of tumors.

In the exploration for therapeutic strategies for tumor, oncolytic viruses have recently displayed potential and effective antitumor effects. ${ }^{10,11}$ For example, there is a novel approach to glioma therapy using an oncolytic adenovirus with two specific promoters. ${ }^{12}$ An oncolytic adenovirus enhances antiangiogenic and antitumoral effects of a replication-deficient adenovirus encoding endostatin by rescuing its selective replication in nasopharyngeal carcinoma cells. ${ }^{13}$ Oncolytic adenovirus expressing IL18 induces significant antitumor effects against melanoma in mice through inhibition of angiogenesis. ${ }^{14}$

Recently found, SNHG1 lncRNA is located at chromosome 11q12.3. SNHG1 has been demonstrated to acts as an oncogene incolon cancer, ${ }^{15-20}$ hepatocellular carcinoma, lung cancer, osteosarcoma, neuroblastoma, and cholangiocarcinoma. However, until now, no report has revealed the function of SNHG1 in the progression and development of OSCC.

In this study, we found that $S N H G 1$ was remarkably increased in OSCC tissue and associated with tumor progression. Moreover, we found that oncolytic adenovirus H101 was more suitable for therapy of OSCC with high SNHG1 expression and chemotherapy more suitable for therapy of OSCC with low SNHG1 expression. As such, SNHG1 can act as a diagnostic biomarker for OSCC and might be a biomarker for treatment options.

\section{Methods}

\section{Clinical Samples}

A total of 152 FFPE block samples consisting of 76 paraffin blocks of OSCC tissue and 76 paraffin blocks of nontumoral tissue collected from the Department of Outpatient Stomatology, Heilongjiang Hospital were used. None of the patients had received chemotherapy or radiotherapy before surgery. This study was approved by the Ethics Committee of Heilongjiang Hospital. All patients provided written informed consent in accordance with the ethical guidelines of Heilongjiang Hospital, and the research conformed with good clinical practice and the principles laid out in the Declaration of Helsinki.

\section{Cell Culture}

The OSCC cell lines SCC9, SCC25, HN4, and Cal27 and the hNOK cell line were purchased from the Shanghai Model Cell Bank (http://www.cellbank.org.cn/mulu.asp). These cells were cultured inDMEM; (Thermo Fisher Scientific, Waltham, MA, USA) containing $10 \% \mathrm{FBS}$, and $100 \mu \mathrm{g} / \mathrm{mL}$ penicillin-streptomycin (BioLight, Shanghai, China) at $37^{\circ} \mathrm{C}$ with $5 \% \mathrm{CO}_{2}$. To knock down $S N H G 1, \mathrm{SCC} 25$ or HN4 cells $\left(2 \times 10^{5}\right)$ were seeded in six-well dishes at 24 hours and then transfected with SNHG1, sh-NC, or sh-SNHG1 per dish using Lipofectamine 3000 according to the manufacturer's instructions. Sequences for sh-SNHG1 (Hanheng Biotechnology) were 5'CCGGCCTTGGGTCTGGAAACTGTTACTCGAGTAACAGTTTCCAGACCCAAGGTTTTTTG-3', and those for shNC (Hanheng Biotechnology) 5'-CCGGATCTCGCTTG GGCGAGAGTAAGTCGACTTACTCTCGCCCAAGCGAGATTTTTTTG-3'.

\section{RNA Extraction and qRT-PCR}

Total RNA was isolated using an RNeasy minikit (Qiagen, Hilden, Germany) in accordance with the manufacturer's instructions. To examine SNHG1 expression, PrimeScript RT master mix (Takara Bio, Kusatsu, Japan) and 2× PCR Master Mix (Thermo Fisher Scientific) were used for reverse transcription (RT) and $\mathrm{qPCR}$, respectively. The relative expression of $S N H G 1$ was calculated and normalized using the $2^{-\Delta \Delta \mathrm{Ct}}$ method. Primer sequences used were: SNHG1 forward 5'-AGGCTGAAGTTACAGGTC3', reverse 5'-TTGGCTCCCAGTGTCTTA-3', GAPDH forward 5'-GGAGCGAGATCCCTCCAAAAT-3, and reverse 5'-GGCTGTTGTCATACTTCTCATGG-3'.

\section{Cell Proliferation}

Equal numbers of transfected OSCC cells $(5,000$ cells/ well) were seeded into a 96-well plate and cell proliferation detected using CCK8 (Biyuntian Biotechnology, Shanghai, China) every 24 hours in accordance with the manufacturer's protocols. A microplate reader was used to examine optical density at $450 \mathrm{~nm}$.

\section{$\mathrm{IC}_{50}$ Assays}

Equal numbers of transfected OSCC cells (5,000 cells/well) were seeded in a 96-well plate and incubated with increasing concentrations of DDP $(0.025,0.05,0.1,0.2,0.4,0.8,1.6$, $3.2,6.4,12.8$, and $25.6 \mu \mathrm{g} / \mathrm{mL})$, Dox $(0.025,0.05,0.1,0.2$, $0.4,0.8,1.6,3.2,6.4,12.8$, and $25.6 \mu \mathrm{g} / \mathrm{mL}), 5 \mathrm{Fu}(0.025$, $0.05,0.1,0.2,0.4,0.8,1.6,3.2,6.4,12.8$, and $25.6 \mu \mathrm{g} / \mathrm{mL})$, 
and H101 $(0.0625,0.125,0.25,0.5,1,5,10,20,40$, and 80 MOI) for another 48 hours. Subsequently, $\mathrm{IC}_{50}$ was detected usingCCK8 (Biyuntian Biotechnology, Shanghai, China) in accordance with the manufacturer's protocols. A microplate reader was used to examine optical density at $490 \mathrm{~nm}$.

\section{Animal Experiments}

To detect OSCC-cell proliferation in vivo, ten BALB/c nude male mice (4-6 weeks old) were purchased from the Shanghai Laboratory Animal Center (Shanghai, China) and divided into two groups (five in each group). $\mathrm{NH}_{4}$-sh-NC $\left(5 \times 10^{6}\right)$ or $\mathrm{NH}_{4}$ sh-SNHG1 cells $\left(5 \times 10^{6}\right)$ were injected subcutaneously into the flanks of nude mice and tumor volumes measured every 7 days according to the formula: $0.5 \times$ length $\times$ width $^{2}$. All animal experiments were performed according to the Guide for the Care and Use of Laboratory Animals and the ethical guidelines of Heilongiiang Hospital. This work was approved by the Ethics Committee of Heilongjiang Hospital.

\section{Statistical Analysis}

GraphPad Prism 6 (GraphPad Software, San Diego, CA, USA) was used for statistical analysis. Differences in SNHG1 expression in OSCC tissue among patients were analyzed using the $\chi 2$ test for the clinical parameters as age, sex, smoking, TNM stage, and lymph-node metastasis. Log-rank (Mantel-Cox) tests, one-way ANOVA, ROC curves, and Student's $t$-tests were used for statistical analysis, and quantitative data are presented as means \pm SD. Results were considered statistically significant at $P<0.05$.

\section{Results}

\section{SNHGI Overexpressed in OSCC Tissue}

Using qRT-PCR, we examined $S N H G 1$ expression in both OSCC and normal tissue. This showed that SNHG1 expression was significantly increased in OSCC tissue compared to normal tissue (Figure 1), which was consistent with previous research, suggesting that aberrant SNHG1 expression might be associated with OSCC progression and development.

\section{Clinical Significance of SNHGI in Indicating OSCC Progression}

Relationships between $S N H G 1$ expression and clinicopathological characteristics of OSCC were studied. As summarized in Table 1, high SNHG1 expression was related to lymph-node metastasis, TNM stage, and tumor size $(P<0.05)$. However, there were no significant relationships

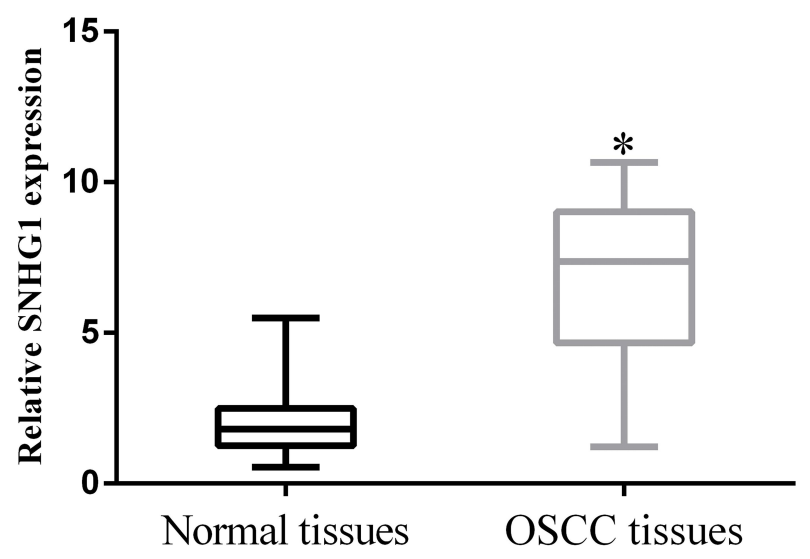

Figure I SNHGI was overexpressed in OSCC tissue $\left(n=76\right.$.) $\left.*_{p}<0.05\right)$. All experiments were repeated three times.

for sex, age, or smoking $(P>0.05)$. Moreover, patients with high $S N H G 1$ expression had poor survival when compared to patients with low SNHG1 expression (Figure 2A), and SNHG1 expression showed potential clinical significance as a tumor biomarker on the ROC curve (Figure 2B).

\section{Knockdown of SNHGI Suppressed Cell Proliferation in OSCC}

To investigate the role of $S N H G 1$ in the progression and development of OSCC, we examined SNHG1 expression

Table I Correlations Between SNHGI Expression and Clinicopathological Characteristics in 76 Patients with OSCC

\begin{tabular}{|c|l|l|l|l|}
\hline & Cases & Low & High & P-value \\
\hline $\begin{array}{c}\text { Age (years) } \\
<60\end{array}$ & $4 I$ & 20 & 21 & 0.623 \\
$\geq 60$ & 35 & 18 & 17 & \\
\hline $\begin{array}{c}\text { Sex } \\
\text { Male } \\
\text { Female }\end{array}$ & 37 & 18 & 19 & 0.715 \\
\hline $\begin{array}{c}\text { Smoking } \\
\text { Never } \\
\text { Quit }\end{array}$ & 36 & 20 & 19 & \\
\hline $\begin{array}{l}\text { TNM stage } \\
\text { I-II } \\
\text { III-IV }\end{array}$ & 40 & 18 & 18 & 0.762 \\
\hline $\begin{array}{l}\text { Tumor size } \\
<5 \text { cm } \\
\geq 5 \mathrm{~cm}\end{array}$ & 35 & 20 & 20 & \\
\hline $\begin{array}{l}\text { LNM status } \\
\text { No } \\
\text { Yes }\end{array}$ & 36 & 13 & 10 & $<0.05$ \\
\hline
\end{tabular}



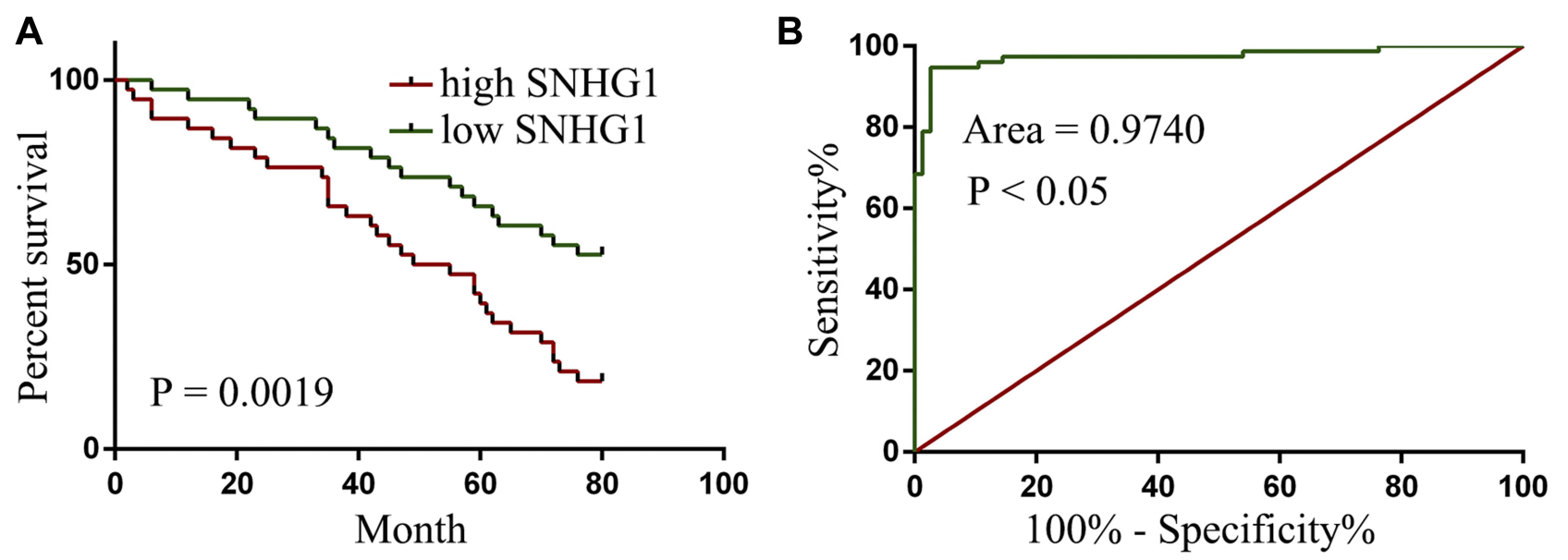

Figure 2 Clinical significance of SNHGI in indicating OSCC progression. (A) Patients with high SNHGI expression had poor survival. (B) SNHGI expression had potential clinical significance as a tumor biomarker based on the ROC) curve $(n=76)$.

in OSCC cells. As shown in Figure 3A, SNHG1 expression was remarkably upregulated in OSCC cells compared to hNOK cells. Moreover, qRT-PCR assays revealed that SNHG1 expression was decreased in OSCC cells transfected with SNHG1 shRNA (Figure 3B), suggesting that we had successfully achieved stable knockdown of SNHG1. CCK8 assays showed that knockdown of SNHG1 significantly inhibited proliferation of OSCC cells (Figure 3C and D). In addition, we found that SNHG1 knockdown markedly suppressed proliferation of OSCC cells in vivo (Figure $3 \mathrm{E}$ ), and mice having received
SNHG1 knockdown had great survival compared to the control group (Figure 3F). Therefore, knockdown of SNHG1 suppressed cell proliferation in OSCC.

\section{High SNHGI Expression in OSCC Suitable for HIOI Therapy}

Oncolytic viruses have recently displayed potentially effective antitumor effects. qRT-PCR assays showed that SNHG1 expression was increased in OSCC cells transfected with SNHG1 and decreased in OSCC cells transfected with sh-SNHG1 (Figure 4A). In addition, CCK8
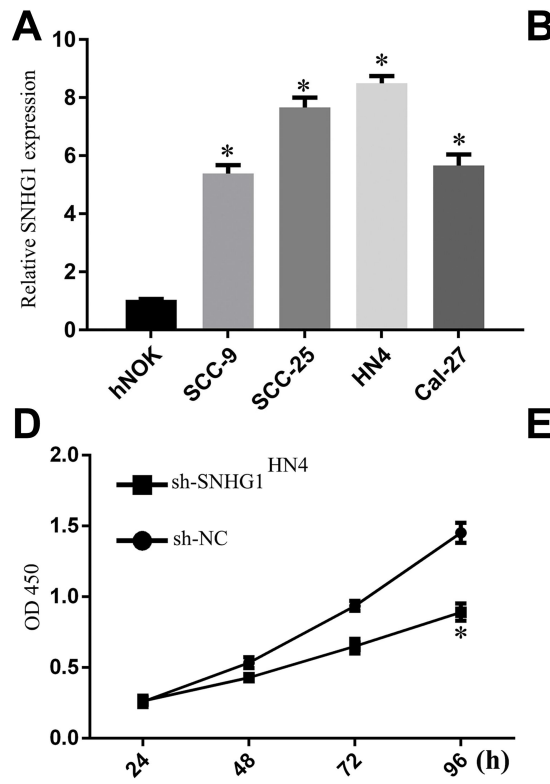

E
B
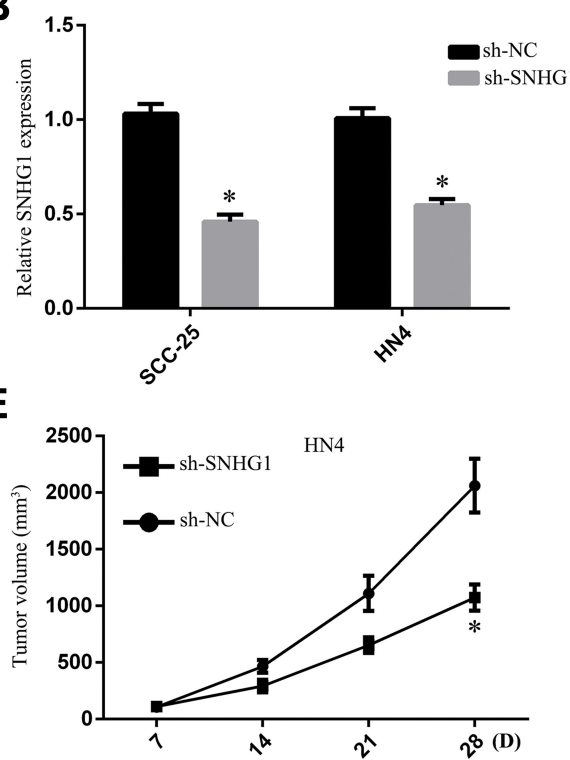

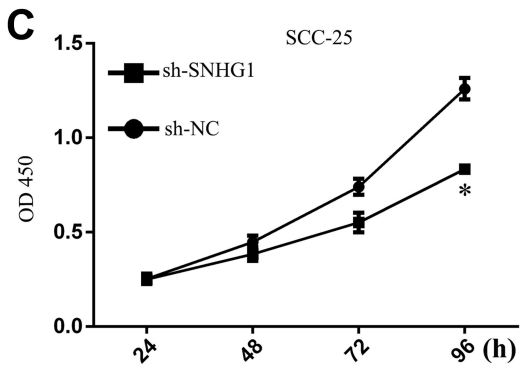

F

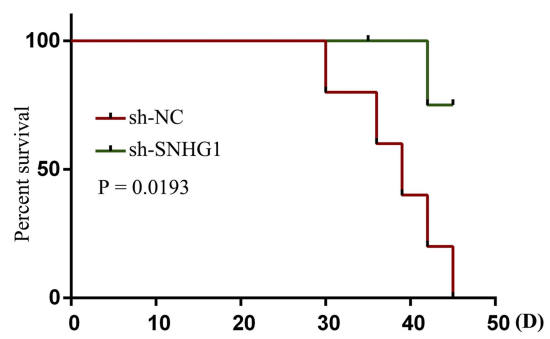

Figure 3 Knockdown of SNHGI suppressed cell proliferation in OSCC. (A) The of SNHGI expression was detected with qRT-PCR assays. (B) Stable knockdown of SNHGI cells was achieved. CCK8 assays showed knockdown of SNHGI expression significantly inhibited cell proliferation in SCC25 (C) and HN4 (D) cells. (E) Tumor volume in mice $(n=5)$. (F) Survival ratio in mice. ${ }^{*} p<0.05$. All experiments were repeated three times. 

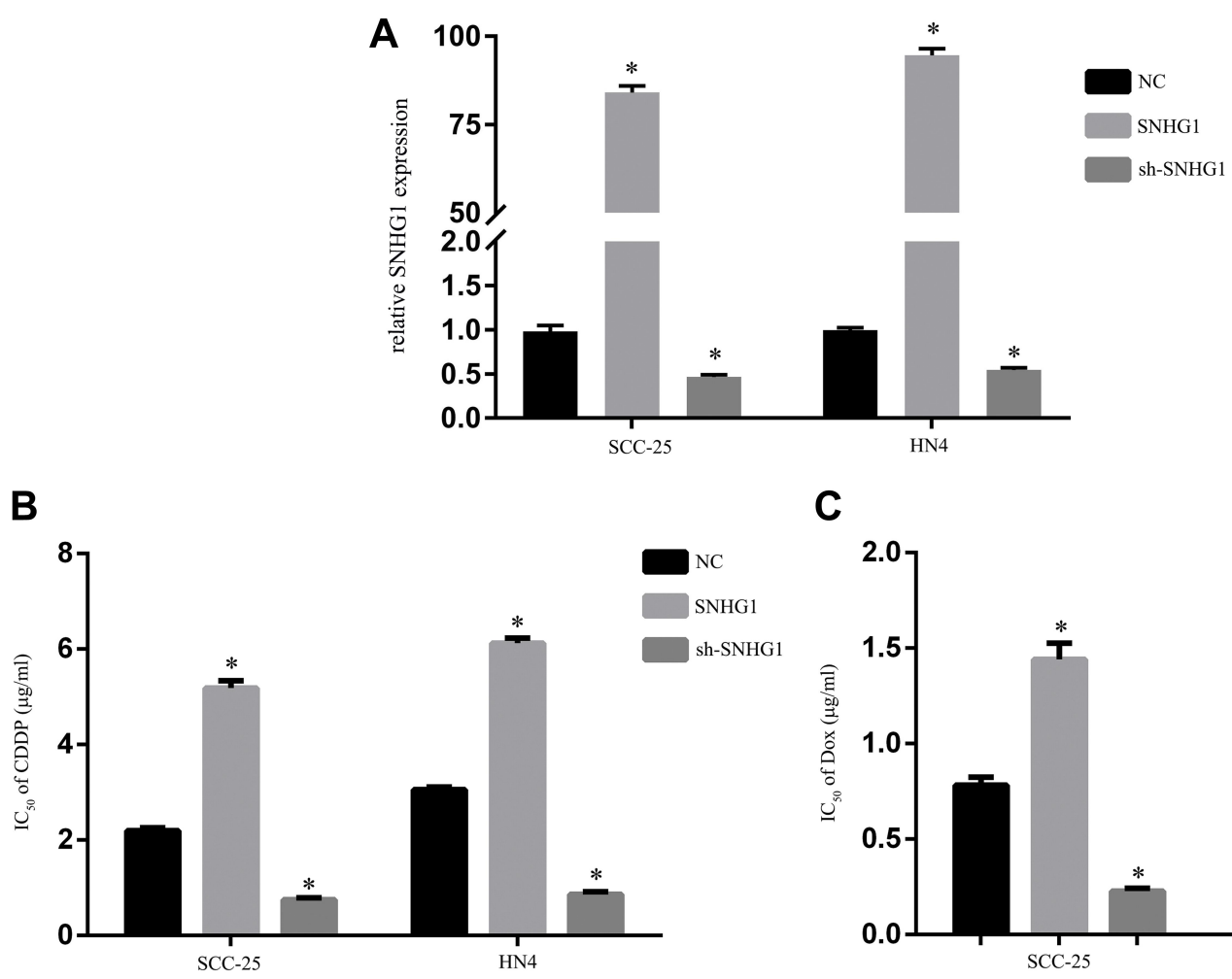

D

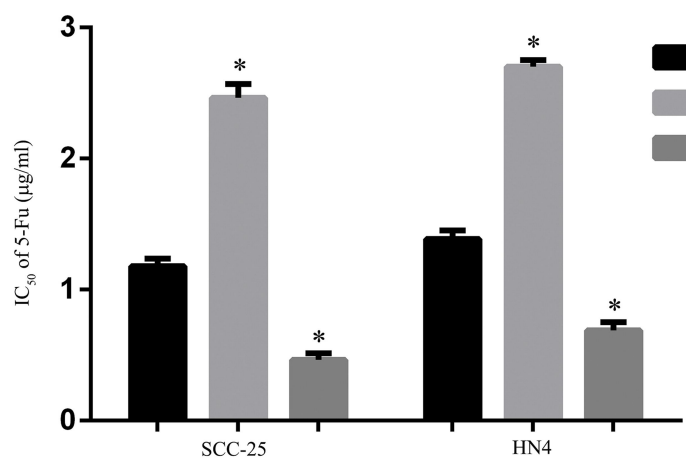

C

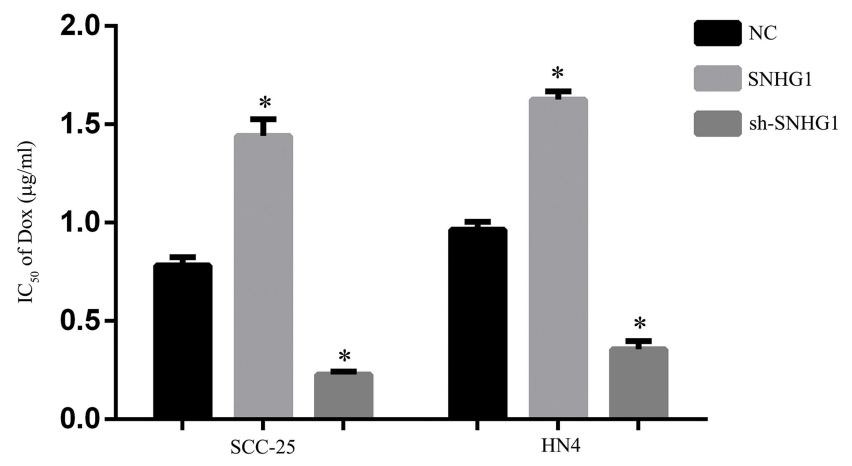

$\mathbf{E}$

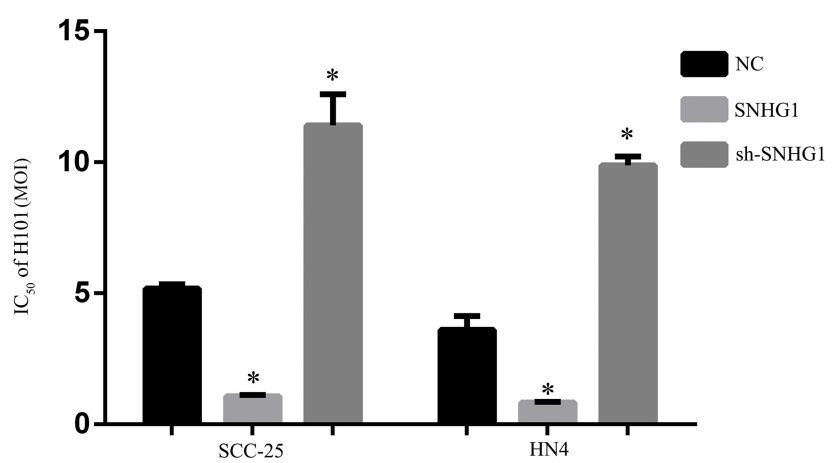

Figure 4 High SNHGI expression in OSCC was suitable for HIOI therapy. (A) SNHGI expression was detected with qRT-PCR assays. IC 50 of cisplatin (B), Dox (C), 5Fu (D), and $\mathrm{HIOI}(\mathbf{E}) .{ }^{*} p<0.05$. All experiments were repeated three times.

assays revealed that $\mathrm{IC}_{50}$ values for cisplatin (Figure 4B), Dox (Figure 4C), and 5Fu (Figure 4D) increased with higher expression of $S N H G 1$ in OSCC cells, while H101 $\mathrm{IC}_{50}$ decreased with higher expression of $S N H G 1$ in OSCC cells (Figure 4E). Therefore, H101 exhibited a stronger antitumor effect in OSCC cells with high SNHG1 expression, and chemotherapy exhibited a stronger antitumor effect in OSCC cells with low SNHG1 expression.

\section{Discussion}

Emerging lines of evidence indicate that lncRNAs function as oncogenes or tumor-suppressor genes to regulate carcinogenesis, and that they can be used as diagnostic or prognostic markers. ${ }^{21}$ For example, a new tumor suppressor - ADAMTS9-AS2 IncRNA - is regulated by DNMT1 and inhibits migration of glioma cells. ${ }^{22}$-FEZF1-AS1 lncRNA promotes tumor proliferation and metastasis in colorectal cancer by regulating PKM2 signaling. ${ }^{23}$ LncRNA-135528 inhibits tumor progression by upregulating CXCL10 through the JAK-STAT pathway. ${ }^{24}$ In this work, the $S N H G 1$ expression was remarkably upregulated in OSCC cells and tissue, and its overexpression was associated with advanced stage and unfavorable prognosis of OSCC patients. Moreover, $S N H G 1$ knockdown suppressed cell proliferation in vitro 
and in vivo. Similarly, SNHG1 lncRNA indicates a poor prognosis and promotes colon cancer tumorigenesis. ${ }^{15}$ Likewise, SNHG1 lncRNA indicates a poor prognosis and promotes hepatocellular carcinoma tumorigenesis. ${ }^{16}$ Upregulated lncRNA SNHG1 contributes to progression of non-small cell lung cancer through inhibition of miR101-3p and activation of the Wnt- $\beta$-catenin signaling pathway. ${ }^{17} \mathrm{As}$ such, our data indicated the oncogenic role of SNHG1 in the development of OSCC.

How to choose a treatment suitable for OSCC is still unclear. Increasingly, studies have indicated that oncolytic viruses have potential and effective antitumor effects. ${ }^{10,11}$ Chemotherapy is commonly used for OSCC treatment; however, the emergence of chemoresistance limits its long-term curative effect. IncRNA plays a significant role in OSCC chemoresistance. ${ }^{25,26}$ Therefore, we ran $\mathrm{IC}_{50}$ assays to examine the sensitivity of oncolytic adenoviruses and chemotherapy drugs to OSCC so as to determine the role of SNHG1 in tumor treatment. We found that oncolytic adenovirus H101 exhibited higher sensitivity in OSCC with high SNHG1 expression and chemotherapy exhibited higher sensitivity in OSCC with low SNHG1 expression. The occurrence and development of tumors involve multiple genes, so in the process of treatment, the selection of highly targeted treatment strategies can achieve more effective therapy. We have identified $S N H G 1$ as a biomarker for OSCC therapy, and our research may help to establish a therapeutic strategies by detecting of SNHG1 expression.

In summary, this research identified the expression and functional role of $S N H G 1$ in OSCC. Our results revealed that SNHG1 was remarkably increased in OSCC tissue and cells. SNHG1 was associated with advanced stage and unfavorable prognosis of OSCC patients, and thus acted as an oncogenic in OSCC. Moreover, knockdown of SNHG1 suppressed cell proliferation in vitro and in vivo. Importantly, we found that oncolytic adenovirus H101 was more suitable for OSCC therapy with high $S N H G 1$ expression and chemotherapy more suitable OSCC therapy with low SNHG1 expression. In future, we will further study the relationship between the expression of SNHG1 in serum and chemotherapy and oncolytic virus therapy to ensure that the OSCC-treatment process can provide faster and more effective treatment options. SNHG1 can act as a diagnostic biomarker for OSCC, and may be expected to be a biomarker for treatment options.

\section{Acknowledgments}

This study was supported by the Heilongjiang Natural Science Foundation (Face project; H2017042).

\section{Disclosure}

The authors declare no conflicts of interest.

\section{References}

1. Warnakulasuriya S. Global epidemiology of oral and oropharyngeal cancer. Oral Oncol. 2009;45(4-5):309-316. doi:10.1016/j. oraloncology.2008.06.002

2. Kademani D. Oral cancer. Mayo Clin Proc. 2007;82(7):878-887. doi:10.4065/82.7.878

3. Neville BW, Day TA. Oral cancer and precancerous lesions. $C A$ Cancer J Clin. 2002;52(4):195-215.

4. Vaishampayan UN, Podgorski I, Heilbrun LK, et al. Biomarkers and bone imaging dynamics associated with clinical outcomes of oral cabozantinib therapy in metastatic castrate-resistant prostate cancer. Clin Cancer Res. 2019;25(2):652-662. doi:10.1158/1078-0432.CCR-18-1473

5. Hsieh MY, Chen G, Chang DC, Chien SY, Chen MK. The impact of metronomic adjuvant chemotherapy in patients with advanced oral cancer. Ann Surg Oncol. 2018;25(7):2091-2097.

6. Hoffman HT, Porter K, Karnell LH, et al. Laryngeal cancer in the United States: changes in demographics, patterns of care, and survival. Laryngoscope. 2006;116(9Pt 2 Suppl 111):1-13. doi:10.1097/01. mlg.0000236095.97947.26

7. Jiang N, Wang X, Xie X, et al. IncRNA DANCR promotes tumor progression and cancer stemness features in osteosarcoma by upregulating AXL via miR-33a-5p inhibition. Cancer Lett. 2017;405:4655. doi:10.1016/j.canlet.2017.06.009

8. Zhang PF, Wang F, Wu J, et al. LncRNA SNHG3 induces EMT and sorafenib resistance by modulating the miR-128/CD151 pathway in hepatocellular carcinoma. J Cell Physiol. 2019;234(3):2788-2794. doi: $10.1002 /$ jcp. 27095

9. Han P, Li JW, Zhang BM, et al. The IncRNA CRNDE promotes colorectal cancer cell proliferation and chemoresistance via miR181a-5p-mediated regulation of $\mathrm{Wnt} /$ beta-catenin signaling. Mol Cancer. 2017;16(1):9. doi:10.1186/s12943-017-0583-1

10. Nemunaitis J, Edelman J. Selectively replicating viral vectors. Cancer Gene Ther. 2002;9(12):987-1000.

11. Geoerger B, Vassal G, Opolon P, et al. Oncolytic activity of p53expressing conditionally replicative adenovirus AdDelta24-p53 against human malignant glioma. Cancer Res. 2004;64(16):57535759. doi:10.1158/0008-5472.CAN-04-0499

12. Liu F, Xu K, Yang H, et al. A novel approach to glioma therapy using an oncolytic adenovirus with two specific promoters. Oncol Lett. 2018;15(3):3362-3368.

13. Liu RY, Zhou L, Zhang YL, et al. An oncolytic adenovirus enhances antiangiogenic and antitumoral effects of a replication-deficient adenovirus encoding endostatin by rescuing its selective replication in nasopharyngeal carcinoma cells. Biochem Biophys Res Commun. 2013;442(3-4):171-176. doi:10.1016/j.bbrc.2013.11.047

14. Zheng JN, Pei DS, Mao LJ, et al. Oncolytic adenovirus expressing interleukin-18 induces significant antitumor effects against melanoma in mice through inhibition of angiogenesis. Cancer Gene Ther. 2010;17(1):28-36. doi:10.1038/cgt.2009.38

15. Yang H, Wang S, Kang YJ, et al. Long non-coding RNA SNHG1 predicts a poor prognosis and promotes colon cancer tumorigenesis. Oncol Rep. 2018;40(1):261-271.

16. Zhang M, Wang $\mathrm{W}, \mathrm{Li} \mathrm{T}$, et al. Long noncoding RNA SNHG1 predicts a poor prognosis and promotes hepatocellular carcinoma tumorigenesis. Biomed Pharmacother. 2016;80:73-79. doi:10.1016/ j.biopha.2016.02.036

17. Cui Y, Zhang F, Zhu C, Geng L, Tian T, Liu H. Upregulated IncRNA SNHG1 contributes to progression of non-small cell lung cancer through inhibition of miR-101-3p and activation of Wnt/beta-catenin signaling pathway. Oncotarget. 2017;8(11):17785-17794. doi:10.18 632/oncotarget. 14854 
18. Wang J, Cao L, Wu J, Wang Q. Long non-coding RNA SNHG1 regulates NOB1 expression by sponging miR-326 and promotes tumorigenesis in osteosarcoma. Int J Oncol. 2018;52(1):77-88.

19. Sahu D, Hsu CL, Lin CC, et al. Co-expression analysis identifies long noncoding RNA SNHG1 as a novel predictor for event-free survival in neuroblastoma. Oncotarget. 2016;7(36):58022-58037. doi:10.18 632/oncotarget.11158

20. Yu Y, Zhang M, Wang N, et al. Epigenetic silencing of tumor suppressor gene CDKN1A by oncogenic long non-coding RNA SNHG1 in cholangiocarcinoma. Cell Death Dis. 2018;9(7):746. doi:10.1038/s41419-018-0768-6

21. Chandra Gupta S, Nandan Tripathi Y. Potential of long non-coding RNAs in cancer patients: from biomarkers to therapeutic targets. Int $J$ Cancer. 2017;140(9):1955-1967.

22. Yao J, Zhou B, Zhang J, et al. A new tumor suppressor LncRNA ADAMTS9-AS2 is regulated by DNMT1 and inhibits migration of glioma cells. Tumour Biol. 2014;35(8):7935-7944. doi:10.1007/ s13277-014-1949-2
23. Bian Z, Zhang J, Li M, et al. LncRNA-FEZF1-AS1 promotes tumor proliferation and metastasis in colorectal cancer by regulating PKM2 signaling. Clin Cancer Res. 2018;24(19):4808-4819. doi:10.1158/ 1078-0432.CCR-17-2967

24. Wang P, Peng X, Zhang J, et al. LncRNA-135528 inhibits tumor progression by up-regulating CXCL10 through the JAK/STAT pathway. Apoptosis. 2018;23(11-12):651-666. doi:10.1007/s10495-0181482-7

25. Fang Z, Zhao J, Xie W, Sun Q, Wang H, Qiao B. LncRNA UCA1 promotes proliferation and cisplatin resistance of oral squamous cell carcinoma by suppressing miR-184 expression. Cancer Med. 2017;6 (12):2897-2908. doi:10.1002/cam4.1253

26. Wang F, Ji X, Wang J, et al. LncRNA PVT1 enhances proliferation and cisplatin resistance via regulating miR-194-5p/HIF 1a axis in oral squamous cell carcinoma. Onco Targets Ther. 2020;13:243-252. doi:10.2147/OTT.S232405

\section{Publish your work in this journal}

OncoTargets and Therapy is an international, peer-reviewed, open access journal focusing on the pathological basis of all cancers, potential targets for therapy and treatment protocols employed to improve the management of cancer patients. The journal also focuses on the impact of management programs and new therapeutic agents and protocols on patient perspectives such as quality of life, adherence and satisfaction. The manuscript management system is completely online and includes a very quick and fair peer-review system, which is all easy to use. Visit http://www.dovepress.com/ testimonials.php to read real quotes from published authors. 